\title{
Seismic modelling of OB stars
}

\section{Marc-Antoine Dupret ${ }^{1}$, Mélanie Godart ${ }^{1}$, Kevin Belkacem ${ }^{1}$ and Arlette Noels ${ }^{1}$}

\author{
${ }^{1}$ Institut d'Astrophysique, Géophysique et Océanographie de l'Université de Liège, \\ Allée du 6 Août 17, 4000 Liège, Belgium \\ email: MA.Dupret@ulg.ac.be
}

\begin{abstract}
A review of the ability of asteroseismology to probe the internal physics of OB stars is presented. The main constraints that can be obtained from the frequency spectrum in pand g-modes pulsators are discussed. Next, we consider energetic aspects of the pulsations in OB stars and show how such study also allows to constrain their internal physics. The cases of p-mixed modes ( $\beta$ Cep stars), g-modes (SPB stars), strange modes and stochastically excited modes are considered.
\end{abstract}

Keywords. stars: evolution, stars: interiors, stars: oscillations, stars: rotation, stars: variables: other, turbulence, convection

\section{Uncertainties in stellar physics}

It is useful to begin with recalling some important problems in stellar physics for which observational constraints can be obtained from asteroseimology.

\subsection{Convective overshoot and penetration}

In all main-sequence massive stars, a convective core is present as a consequence of the high temperature dependence of the CNO cycle. The region just above this core is important and poorly known in these stars. A key ability of asteroseismology is to probe it (Noels et al. 2010). Because of their inertia, convective elements penetrate slightly in the subadiabatic layers just above the convective core. As the Peclet number is very high there, we may assume that these plumes keep their heat content as they penetrate in these regions. Hence, the buoyancy braking is large and the plumes cool down the gas around them. (Zahn 1991) proposes to call this process "convective penetration", in contrast with the small Peclet numbers cases called "convective overshoot". We can expect that this cooling increases the temperature gradient until it becomes nearly adiabatic. This decreases significantly the braking and the plumes can penetrate further. The extension and description of this extra-mixed overshooting region is thus very uncertain. Its size is generally parametrized as a fraction of the pressure scale height : $\Delta r_{o v}=\alpha_{o v}|d r / d \ln P|$, where $\alpha_{o v}$ is the so-called overshooting parameter. It must be stressed that stellar evolution strongly depends on the extension of this extra-mixing which continuously transports fresh material into the core where it is burned.

\subsection{Semi-convection}

One of the following two criteria is generally used for the determination of the convective core boundary. On the one hand, the Schwartzschild criterion $\nabla_{\text {rad }}=\nabla_{a d}$ assumes thermal neutrality (null convective heat transfer) at the boundary $\left(\nabla_{\text {rad }} \equiv\right.$ $\left.3 \kappa P L /\left(16 \pi a c G m T^{4}\right), \nabla_{a d} \equiv \partial \ln T /\left.\partial \ln P\right|_{s}\right)$. On the other hand, the Ledoux criterion $\nabla_{L}=\nabla_{a d}$ assumes dynamical neutrality (null force) at the boundary $\left(\nabla_{L} \equiv \nabla_{\text {rad }}-\frac{\phi}{\delta} \nabla_{\mu}\right.$ 
with $\left.\nabla_{\mu} \equiv d \ln \mu / d \ln P\right)$. In massive stars, the convective core mass sometimes grows with time or decreases slowly. In principle, this should lead to a discontinuity of chemical composition at the boundary of the convective core. However, with the assumption of full mixing below and no mixing above, it appears impossible to define consistently this boundary. The only solution is to assume partial mixing in a so-called semi-convective zone above the convective core. In this semi-convective region, $\nabla_{a d} \leqslant \nabla \leqslant \nabla_{L}(\nabla \equiv d \ln T / d \ln P$ is the "real gradient" corresponding to the temperature stratification in the star), but the exact temperature and chemical stratifications remain quite uncertain. Key constraints on this region could be obtained from asteroseismology (Noels et al. 2010).

\subsection{Microscopic diffusion}

Microscopic chemical transport is certainly present in massive stars under the action of two forces: the gravitation and the radiative forces (impulsion of photons transmitted to the gas). The gravitation force is proportional to the mass so that the heaviest particles are pushed down. The absorption of photons varies strongly from one type of particle to another. Particles with high cross section in the frequency range around the maximum of the Planck function are strongly pushed up. This is important in massive stars where radiation pressure is significant. The action of these forces implies a chemical transport in stellar interiors under the form of a microscopic diffusive process. However, significant uncertainties remain associated to these processes (collision integrals, shielding, ...).

\subsection{Macroscopic chemical and angular momentum transfer}

As detailed by Zahn (these proceedings), rotation is at the origin of two macroscopic transfer processes of very distinct natures in radiative zones. First, the Von Zeipel theorem shows that rotation must generate a meridional circulation. After a transient phase, this circulation settles in a quasi-stationary regime allowing to advect angular momentum (and at the same time chemicals) towards the surface where it is lost for example by winds. Second, the horizontal shear and other instabilities due to differential rotation generate turbulence. This turbulence is expected to be much more vigorous in the horizontal plane because of the stabilizing effect of buoyancy, justifying the so-called shellular approximation where differential rotation in latitude is assumed negligible. Turbulence in radiative zones acts as a diffusive transfer mechanism, which is qualitatively very different from the advection by meridional circulation. The main source of uncertainties lies in the turbulent diffusion coefficients that must be used to model this process (Mathis et al. 2004).

Another transfer mechanism that was shown to be important these last years comes from gravity waves and modes. Progressive waves emitted at the boundary of convective zones transfer angular momentum in the radiative zone where they are dissipated. Works of Charbonnel \& Talon (2005) show that this mechanism is able to explain the solid body rotation in the solar radiation zone. It should not be forgotten that another possibly important mechanism in massive stars is the transfer by stationary gravity modes. This transfer process is expected to weaken the differential rotation caused by evolutionary core contraction (Lee \& Saio 1993). Finally, we mention that magnetic field and differential rotations certainly influence each other, we refer to Zahn (these proceedings) for more details. For a general review of all these processes, see e.g. Talon (2008).

\subsection{Opacities}

Many stellar physics problems of the 20th century were solved thanks to improvements in the computations of opacities. The last important one was the explanation of the driving of $\beta$ Cep stars thanks to the OPAL opacity tables. It is clear that the determination of 
precise opacities is not yet a solved problem. Asteroseismology and more specifically nonadiabatic asteroseismology (see Sect. 3.2) give important constraints on the opacities, indicating that models with the current best opacities: OP (Seaton 2005) and OPAL (Rogers \& Iglesias 1992) are not yet able to explain some observations.

\section{Interpreting the frequencies}

We first recall that there are mainly two types of pulsation modes: the pressure (or p-) modes similar to acoustic standing waves and the gravity (or g-) modes in which the restoring force is the buoyancy. These two families are not exclusive, many times in intermediate and high mass stars, we also encounter the so-called mixed modes that have at the same time a gravity mode nature in the core and a pressure mode nature in the envelope of the star.

\subsection{Seismic probe by pressure and mixed modes}

The frequency spectrum of pressure modes mainly depends on the sound speed profile $c(r)$ in the envelope of the star. Its square is given by:

$$
c^{2}=\Gamma_{1} P / \rho .
$$

As in an ideal gas, $P / \rho \propto T / \mu$, we see that probing the sound speed means essentially probing the temperature and the internal chemical composition throughout $\mu$ (mean molecular weight) and $\Gamma_{1} \equiv \partial \ln P /\left.\partial \ln \rho\right|_{s}$. Probing this sound speed was already achieved with high precision for the Sun by helioseismology. Asteroseismology aims to do the same for other stars, but with less precision since the number of observed modes is by far lower.

In the context of OB stars, $\mathrm{p}$-mixed modes pulsations are observed in $\beta$ Cep stars. It is important to emphasize that such oscillations are very different from the p-modes oscillations observed in the Sun and other solar-like stars. Solar-like oscillations correspond to high order p-modes near the asymptotic regime. As a consequence, their frequency patterns are regular, mode identification is "easy", but the number of individual constraints is reduced by the asymptotic degeneracy. On the contrary, each p-mixed mode in a $\beta$ Cep star probes a very different region of the star (see for example the kernels illustrated in Fig. 5 of Pamyatnykh et al. 2004). That means that each frequency gives a completely independent constraint on the stellar interior model. Even with few modes, key informations can be obtained, if the modes are identified. Several examples illustrate the power of asteroseismology applied to $\beta$ Cep stars: for example $\nu$ Eri (Pamyatnykh et al. 2004; Ausseloos et al. 2004; Dziembowski \& Pamyatnykh 2008) and HD 129929 (Aerts et al. 2003; Dupret et al. 2004). The main unknown global parameters of a massive star are its mass, age, initial hydrogen fraction $X$, heavy elements fraction $Z$ and the overshooting parameter. Hence, with five identified modes of different $(\ell, n)$, it is possible to determine them with precision. With constraints on effective temperature, gravity and metallicity from spectroscopy or photometry, this minimum required number of identified modes can be reduced. Non-adiabatic analysis also gives additional constraints (see Sect. 3.2). Best constrained cases such as $\nu$ Eri show that standard models are not able to fully reproduce the observations.

For the same reason (mixed nature of the modes), the kernels associated to rotation show that each multiplet of a $\beta$ Cep star probes the rotation rate in a distinct region (see Fig. 6 in Pamyatnykh et al. 2004 and Fig. 7 in Dupret et al. 2004). With two multiplets, it is already possible to test if solid body rotation is compatible with seismic observations. The results differ from a particular star to another: in $\nu$ Eri and HD 129929, 
asteroseismology shows that the core rotates about three times faster than the envelope. But in $\theta$ Oph (Briquet et al. 2007), solid rotation is compatible with seismic observations. Hence the question arises: which physical processes act differently in stars at the same location in the HR diagram, producing solid rotation in one case and strong differential rotation in another. Maybe, the answer could come from the magnetic field ? Indeed, the freezing effect of the magnetic field is well known and, if strong enough, this could produce solid body rotation. Unfortunately, no magnetic field has been detected in $\theta$ Op (Hubrig et al. 2006). We mentioned above the possibility of angular momentum transfer by gravity modes. Depending on the pulsational history of a star, angular momentum transfer could be significant in some of them, leading to solid body rotation; while this transfer could be weaker in others, letting evolutionary core contraction produce the differential rotation. However, quantitative models and simulations are needed to confirm this possibility.

Macroscopic transfer of angular momentum and chemicals by differential rotation could also be constrained from the frequencies of identified p-mixed modes. Indeed, Montalbàn et al. (2008) showed that the frequencies of p-mixed modes in $\beta$ Cep models are significantly affected by turbulent mixing. Seismic constraints on these processes for particular stars are expected to be obtained in the near future.

\subsection{Seismic probe by high order gravity modes}

The period spectrum of gravity modes mainly depends on the so-called Brunt-Vaïsälä (BV) frequency, also called Buoyancy frequency. Its square is defined by:

$$
N^{2}=g\left(\frac{1}{\Gamma_{1}} \frac{d \ln P}{d r}-\frac{d \ln \rho}{d r}\right) .
$$

We have approximately (fully ionized ideal gaz) :

$$
N^{2} \simeq \frac{g^{2} \rho}{P}\left(\nabla_{a d}-\nabla+\nabla_{\mu}\right),
$$

where $\nabla_{\mu} \equiv d \ln \mu / d \ln P$ is the " $\mu$-gradient" corresponding to the molecular weight variation throughout the star. Fine details of the internal structure of stars appear throughout $\nabla$ and $\nabla_{\mu}$ and can be probed by gravity modes (if observed).

In the asymptotic limit, the periods of g-modes are approximately given by the following law:

$$
P_{k}=\frac{\pi^{2}\left(2 k+n_{e}\right)}{\sqrt{\ell(\ell+1)} \int_{r_{0}}^{R}|N| / r d r},
$$

where $r_{0}$ is the radius of the convective core. The integral of the BV frequency appearing in the denominator is thus the main physical quantity that can be deduced from the g-modes spectrum, simply by considering the period spacing between consecutive modes. Typically, as the star evolves, the density contrast between the core and the envelope increases, therefore the BV frequency increases in the core and the period spacing decreases. Of course, information more useful than the mean period spacing can be potentially extracted from the g-modes spectrum. Let's consider the typical case of a main-sequence star with a receding convective core letting behind it a region of variable molecular weight. According to Eq. (2.3), the resulting strong molecular weight gradient produces a peak in the BV frequency. In particular, a strong discontinuity of $N$ is predicted at the top of this $\mu$-gradient region. The effect of such discontinuity on the g-mode period spacing can be determined. As shown e.g. in Miglio et al. (2008), instead of being perfectly constant as in Eq. (2.4), this period spacing $\Delta P(k)=P_{k}-P_{k-1}$ oscillates with 
a "wavelength":

$$
\Delta k \simeq \frac{\int_{r_{0}}^{R}|N| / r d r}{\int_{r_{0}}^{r_{1}}|N| / r d r}
$$

where $r_{1}$ corresponds to the location of the discontinuity. For a ZAMS model, there is not yet a $\mu$-gradient region, thus $\Delta k \rightarrow \infty$ and the period spacing is independent of $k$. As the star evolves, the $\mu$-gradient region increases in size and thus the denominator of Eq. (2.5) increases. Hence, an oscillation of $\Delta P(k)$ appears with a "wavelength" $\Delta k$ which decreases progressively. This is illustrated for example in Fig. 16 of Miglio et al. (2008). Hence, if such oscillation of the period spacing is observed, it allows to determine accurately the evolutionary state of a star. More quantitatively, $\Delta k$ is a precise indicator of the central hydrogen abundance $X_{c}$ of a MS star.

What about overshooting now ? Miglio et al. (2008) showed that, for massive stars with fixed $X_{c}, \int_{r_{0}}^{r_{1}}(N / r) d r$ and thus $\Delta k$ do not depend significantly on the amount of overshooting (assuming instantaneous mixing). This is different for intermediate mass stars such as $\gamma$ Dor stars where the nuclear burning region is slightly larger than the convective core. But this does not mean that overshooting cannot be constrained from the g-modes periods in massive stars. Stellar evolution tracks strongly depend on overshooting. If, for example, the effective temperature, gravity and metallicity of the star are determined with enough precision, combined seismic and non-seismic constraints could allow us to constrain overshooting.

Finally, a very important ability of g-modes is to probe the $\mu$-gradient region and thus chemical transfer mechanisms due for example to differential rotation. We have seen above that the discontinuity of $\nabla_{\mu}$ at the top of this region leads to an oscillation of $\Delta P(k)$. Macroscopic chemical transfer mechanisms such as turbulent diffusion smooth this discontinuity (see Fig. 22 of Miglio et al. 2008). As a consequence, the amplitude of the oscillation of $\Delta P(k)$ is smaller and decreases with $k$. With high turbulent diffusivity, $\Delta P$ becomes independent of $k$. A clear signature of diffusive mixing can thus be detected in the period spacing.

A nice application of g-modes asteroseismology was recently possible thanks to the detection of hundreds of modes in the SPB HD 50230 by CoRoT (Degroote et al. 2010a). The very important discovery was the detection of 8 consecutive g-modes showing a clear oscillation in the function $\Delta P(P)$. Based on these observations, seismic modelling was possible. The main results are that $60 \%$ of the central hydrogen has already been consumed, the overshooting parameter is larger than 0.2 and the small amplitude of the deviation to constant spacing indicates a smooth gradient of chemical composition above the convective core incompatible with instantaneous mixing.

\subsection{Mode identification}

An important problem in asteroseismology is the mode identification. In the asymptotic limit, the spectrum of frequencies is expected to be regular. We already mentioned the quasi-constant period spacing predicted for high order gravity modes. In high order pressure modes, quasi-equidistant frequency separation is expected, according to the following approximate relation:

$$
\nu_{n, \ell} \simeq\left(n+\frac{\ell}{2}+\frac{1}{4}+\alpha\right) \Delta \nu-(A \ell(\ell+1)-\delta) \frac{\Delta \nu^{2}}{\nu_{n, \ell}}-m\left(1-C_{n, \ell}\right) f_{\mathrm{rot}}
$$

where $\Delta \nu$ is called the large separation. Such regularities are easy to detect in solar-type oscillations. They allow us to identify the modes, which makes seismic modelling possible without need of additional informations. 
However, such regularities are not present in low-order modes such as those observed in $\beta$ Cep and $\delta$ Sct stars, because of their mixed character. Mode identification is thus impossible on the basis of the frequencies alone. Two types of additional observables can be used for mode identification. First, we have the spectroscopic line-profile variations. They result from the pulsational surface velocity field (Doppler effect) and allow us to constrain it. In particular, their interpretation allows us to determine the degree $\ell$ and azimuthal order $m$ of the modes, see e.g. the review of Telting (2008) and references therein. Secondly, we have the photometric amplitudes and phase differences of magnitude variations in different passbands. They depend on the degree $\ell$ of the modes, so that it can be determined by comparing theoretical predictions with observations. The basics of this method are detailed in Watson (1988). Several improvements have been proposed to take non-adiabatic and rotational effects into account, see Handler (2008) for a review.

\subsection{Fast rotation}

All previous discussions were valid for slow rotators. In fast rotators such as Be stars, seismic modelling is much more difficult. Fast rotation has two important effects. Firstly, centrifugal force breaks down the spherical symetry when $\Omega^{2} R^{3} /(G M)$ is significant. Secondly, the Coriolis force plays a large role in the mode dynamics when $\Omega / \sigma$ is significant ( $\Omega$ is the rotation rate and $\sigma$ the angular pulsation frequency). Usual modelling tools for slow rotators are evidently not adequate for rapid rotators.

Concerning the internal structure, a new generation of full 2D stellar models are needed. The most rigorous approach would be to develop 2D stellar evolution codes fully including the deformation due to centrifugal force and the transfer processes associated with differential rotation. Works in this direction are being done with spectral (Espinosa \& Rieutord 2007) and finite differences (Deupree 1995) numerical approaches; but the task remains hard. A simpler and efficient approach is to use the Self Consistent Field (SCF) method (see e.g. Jackson et al. 2005; Roxburgh 2004 for the most recent papers), which is valid for conservative rotation laws (solid body or cylindrical). It is also useful to mention the "characteristics" method proposed by Roxburgh (2006) which allows us to compute $2 \mathrm{D}$ structure models for arbitrary differential rotation laws. The input of the code are here a $1 \mathrm{D}$ structure model and the rotation law $\Omega(r, \theta)$; the output is a $2 \mathrm{D}$ deformed model with the same mean structure. Computation times are much shorter with such an approach, which would make possible to compute numerous models as required for seismic modelling. Finally, a widely used but approximate approach is to model the structural effect of rotation in a perturbative way: spherical symetric component plus perturbation proportional to $P_{2}(\cos \theta)$.

Let's consider now the modelling of oscillations in rapid rotators. Here also, a new generation of oscillation codes is required. The perturbative approach does not apply when the centrifugal deformation and/or $\Omega / \sigma$ are significant. In these cases, the coupling between oscillations and rotation must be modelled in a non-perturbative way. Several adiabatic oscillation codes have been developed for this purpose: with a finite difference approach (Clement 1998), a full spectral approach (Reese et al. 2006) and a mixed finite difference-spectral approach (Reese et al. 2009, Ouazzani et al. in preparation). Concerning non-adiabatic oscillation codes, the best present models are obtained with the method of Lee \& Baraffe (1995) in which a perturbative approach is adopted for the structure models and a spectral non-perturbative approach is used for the oscillations.

As shown by several studies, the mode geometry is completely different from spherical harmonics in fast rotators (see e.g. Fig. 3 in Reese et al. 2009). The p-modes of fast rotators fall into three categories: island, chaotic and whispering gallery modes (Lignières 
\& Georgeot 2009). The structure of the oscillation frequency spectrum is also completely different from the slow rotation case: in the asymptotic limit, old regularities such as Eq. (2.6) are no longer valid, but new ones appear. Reese et al. (2009) proposed new asymptotic laws which better agree with the frequency patterns in fast rotators.

It is clear that the main price for accurate modelling of fast rotators pulsations is the computation time. Asteroseismology by the direct method generally requires the computation of many structure models and their oscillations in order to find the best fit solution. Such an approach is currently not possible because of computation time. New strategies feasible even with a small number of models are needed (Lovekin \& Goupil 2010).

\section{Energetic aspects of stellar oscillations}

\subsection{Driving mechanism}

The first part of this review considered adiabatic modelling of stellar oscillations. Such an approach is adequate for the determination of the frequencies because the modes mainly propagate in deep enough regions where the oscillations are quasi-adiabatic. But such an approach does not allow us to study the energetic aspects of oscillations such as the driving mechanisms at the origin of the oscillations. Non-adiabatic modelling is required to study these aspects. The main uncertainties in such models concern the time-dependent interaction between convection and oscillations. Fortunately, this difficult aspect does not play a significant role in massive stars. In OB stars, the driving of the modes comes from a standard $\kappa$-mechanism occurring in the iron M-shell opacity bump at $T \approx 2 \times 10^{5} \mathrm{~K}$ (Dziembowski et al. 1993a; 1993b). The main driving or damping of the modes always occurs in the transition zone where the thermal relaxation time is of the order of the pulsation period. In $\beta$ Cep stars, the transition region for low order pressure modes coincides with the iron opacity bump, which explains their efficient driving. In the cooler SPB stars, the iron bump is deeper, coinciding with the transition zone for long period high order gravity modes.

\subsection{Non-adiabatic asteroseismology}

Asteroseismology is generally seen as a method for constraining internal structure of stars by using the information given by the observed pulsation frequencies. However, this is often not possible without mode identification. We call non-adiabatic asteroseismology a method of constraining stellar interiors based on observables which are theoretically determined by non-adiabatic computations. What are these observables ? Firstly, nonadiabatic computations determine which modes of a given model are excited and thus able to grow up to observable amplitudes. The constraint is here obtained by comparing the range of observed frequencies to the predicted range of excited modes. Secondly, we mentioned in Sect. 2.3 the photometric mode identification method. Theoretical determination of the multi-color photometric amplitude ratios and phase differences requires the knowledge of the normalized amplitude and phase of effective temperature variations, which are obtained by non-adiabatic computations. The constraints come here from the comparison between theoretical and observed amplitude ratios and phase differences.

Non-adiabatic predictions in massive stars mainly depend on the opacity $\kappa$ and its derivatives $\partial \ln \kappa /\left.\partial \ln T\right|_{\rho}$ and $\partial \ln \kappa /\left.\partial \ln \rho\right|_{T}$ in the driving region. Non-adiabatic asteroseismology in massive stars gives thus the ability to constrain the opacity. The current most precise opacities are given in the OPAL (Rogers \& Iglesias 1992) and OP (Opacity project) tables. The last improvements in OP and a comparison with OPAL are presented in Badnell et al. (2005). The opacities also strongly depend on the adopted 
mixture. Miglio et al. (2007a) computed instability strips (IS) of $\beta$ Cep and SPB stars with OPAL and OP opacity tables and two different mixtures: Grevesse \& Noels (1993) and the new solar mixture of Asplund et al. (2005). They found significant differences between OP and OPAL IS: mainly the IS of SPB stars obtained with OP extends to hotter stars compared to OPAL. These differences indicate that current uncertainties in opacity computations have a significant observable effect. They also found a significant impact of the metal mixture on the IS: higher overtones are excited with the mixture of Asplund et al. (2005).

How do theory and observations compare? Hybrid pulsators in which both $\beta$ Cep type p-mixed modes and SPB type g-modes are observed are particularly constraining. An important example is $\nu$ Eri in which the range of observed modes is larger than what theory predicts. More modes are excited with OP than with OPAL, but the excitation of the highest overtone is still not obtained (Dziembowski \& Pamyatnykh 2008). Another interesting case is $\gamma$ Peg (Handler et al. 2009).

$\beta$ Cep and SPB candidates were recently discovered in the SMC (Karoff et al. 2008; Diago et al. 2008), which challenges theory. Indeed, the metallicity of SMC is very low $(Z \approx 0.001-0.004)$ so that the iron opacity bump is not strong enough to allow efficient $\kappa$-driving of the modes (Salmon et al. 2009). A significant increase of the opacity in the iron bump would be required to excite $\beta$ Cep and SPB modes in the SMC (Salmon et al. in preparation).

Finally, as above-mentioned, comparison between theoretical and observed multi-color photometric amplitude ratios and phase differences can also be used to constrain the opacity and metallicity (see Daszyńska \& Walczak 2009, Daszyńska \& Walczak 2010 for the most recent studies of this type).

\subsection{Energetic aspects in fast rotators}

We discussed in Sect. 2.4 the effect of fast rotation on the frequencies and mode geometry under the adiabatic approximation. Non-adiabatic non-perturbative pulsation codes would be required to study the excitation and damping mechanisms in fast rotators. Present models include approximations. For example, the traditional approximation is often used for the study of the driving mechanisms (Townsend 2005) and in the nonadiabatic models required for photometric mode identification (Townsend 2003). We recall however that this approximation assumes spherical symetry of the equilibrium model. It is justified for g-modes in slow or moderate rotators but not in fast rotators.

Non-adiabatic models of fast rotators are complex but there is at least one simple effect with significant observational impact. In the energy equation, what matters is the pulsation frequency from the point of view of the corotating frame. The frequency range of excited modes expressed in this frame does not depend strongly on $m$. We have

$$
\sigma_{I}=\sigma_{c}-m \Omega
$$

where $\sigma_{I}$ is the frequency in the observer inertial frame and $\sigma_{c}$ is the frequency in the corotating frame. As a simple consequence of this relation, the excited frequencies in the observer frame strongly depend on $m$. For example, the retrograde $(m>0)$ modes can have very long observed periods. A very interesting extreme case is when $\sigma_{c}<\Omega$, as can be the case for the g-modes of Slowly Pulsating Be (SPBe) stars. From Eq. (3.1), modes with different $m$ are therefore separated in distinct groups. This is excellent news, as it solves the problem of mode identification ! Such an effect was indeed observed in several SPBe stars (Cameron et al. 2008). 


\subsection{Driving non-radial pulsations in post $M S$ B stars}

Post MS stars are characterized by a high density contrast between the core and the envelope. As a consequence, the BV frequency is very large in the radiative core and all non-radial modes behave like g-modes in these deep regions. Theory predicts that the radiative damping of these modes is very large because of their high wavenumber $\left(k_{r}=\sqrt{\ell(\ell+1)} N /(\sigma r)\right)$. Therefore, non-radial modes are not expected to be excited in post MS B stars. The detection of many non-radial modes in a B supergiant by MOST was thus a big surprise, but not for a long time since it could be explained in the paper reporting their discovery by Saio et al. (2007). As a consequence of the evolution of the convective core during the MS phase, the temperature gradient $\nabla$ becomes flat and slightly subadiabatic above it. When the H-burning shell appears during stellar evolution, $L / m$ increases there, the temperature gradient easily becomes superadiabatic, which leads to the appearence of an Intermediate Convection Zone (ICZ) above the H-burning shell. Saio et al. (2007) showed that this ICZ can act as a propagation barrier for the non-radial modes: most are trapped in the radiative core below it, but some others are trapped in the envelope. These last modes have negligible amplitudes in the core and do not undergo significant radiative damping. They are driven by a $\kappa$-mechanism in the Fe opacity bump like in MS SPB stars.

The presence of the ICZ at the origin of these modes depends on several processes. As shown by Godart et al. (2009), mass loss inhibits the appearance of the ICZ; the observation of these mode gives thus an upper limit on the mass loss rate. Overshooting also has such an inhibiting effect, as well as rotational mixing. Finally, depending on the convection criterion (Schwartzschild versus Ledoux), the resulting ICZ is of very different nature (Lebreton et al. 2009). These processes and their consequences can thus be constrained by the observation of non-radial modes in B supergiants.

\subsection{Strange modes}

As a consequence of the combined actions of the L- and M-shell iron opacity bumps and the high radiative pressure, a sound speed inversion can be present in the envelope of massive stars. This produces a cavity where modes can be trapped: the strange modes (Saio et al. 1998). Strange modes are of different kinds: some have an adiabatic counterpart, others are purely thermal. There are also the convective strange modes (Saio, these proceedings): $\mathrm{g}^{-}$modes which are shown to oscillate when non-adiabatic aspects are included. Mode trapping can also occur in the stellar atmosphere when a temperature inversion is present (Godart et al. these proceedings), producing another kind of strange modes. As strange modes propagate in very superficial layers, they hold informations about these regions (sound speed, ....). However, non-linear effects are strong because of their very low inertia. Strange modes could also play a role in mass ejection such as in LBV stars (Glatzel 2009). Finally, we mention the recent and interesting detection by CoRoT (and in spectroscopy) of a mode which could be a strange mode (Aerts et al. 2010).

\subsection{Solar-like modes in massive stars}

High order p-modes have recently been dicovered by CoRoT in a $\beta$ Cep star (Belkacem et al. 2009) and in a O-star (Degroote et al. 2010b). A time-frequency (or wavelet) analysis clearly shows their stochastic nature, which makes them similar to the solar modes. Stochastic excitation models (Belkacem et al. 2010) show that these modes could be excited by the turbulent motions in the iron subsurface convection zone (Cantiello, these proceedings) or near the top of the convective core. The eigenfunction shape in the excitation region significantly affects the observed amplitudes. In the first case (subsurface 
excitation), a modulation of the amplitudes with frequency is predicted. In the second, a decrease of the amplitudes with frequency is predicted, simply because high frequency modes have small normalized amplitudes in the core. Current large uncertainties in the description of these region (convective velocities, eddy-time correlation, ...) also have a significant impact on the predicted amplitudes. The comparison with observations gives thus a unique opportunity to improve the knowledge of these regions. Of course, the frequencies of these modes can also be used to constrain the envelope structure.

\section{References}

Aerts, C., Thoul, A., Daszyńska-Daszkiewicz, J., \& Scuflaire, R. et al. 2003, Science 300, 1926 Aerts, C., Lefever, K., Baglin, A., \& Degroote, P. et al. 2010, A\&A, 513, L11

Asplund, M., Grevesse, N., Sauval, A. J., \& Allende Prieto, C. et al. 2005, A\& A, 431, 693

Ausseloos, M., Scuflaire, R., Thoul, A., \& Aerts, C. 2004, MNRAS, 355, 352

Badnell, N. R., Bautista, M. A., Butler, K., \& Delahaye, F. et al. 2005, MNRAS, 360, 458

Belkacem, K., Samadi, R., Goupil, M.-J., \& Lefèvre, L. et al. 2009, Science, 324, 1540

Belkacem, K., Dupret, M. A., \& Noels, A. 2010, A\& A, 510, A6

Briquet, M., Morel, T., Thoul, A., \& Scuflaire, R. et al. 2007, MNRAS, 381, 1482

Cameron, C., Saio, H., Kuschnig, R., \& Walker, G. A. H. et al. 2008, ApJ, 685, 489

Charbonnel, C. \& Talon, S. 2005, Science, 309, 2189

Clement, M. J. 1998, ApJS, 116, 57

Daszyńska-Daszkiewicz, J. \& Walczak, P. 2009, MNRAS, 398, 1961

Daszyńska-Daszkiewicz, J. \& Walczak, P. 2010, MNRAS, 403, 496

Degroote, P., Aerts, C., Baglin, A., \& Miglio, A. et al. 2010a, Nature, 464, 259

Degroote, P., Briquet, M., Auvergne, M., \& Simón-Díaz, S. et al. 2010b, A\&A, 519A, 38

Deupree, R. G. 1995, ApJ, 439, 357

Diago, P. D., Gutiérrez-Soto, J., Fabregat, J., \& Martayan, C. 2008, A\& A, 480, 179

Dupret, M.-A., Thoul, A., Scuflaire, R., \& Daszyńska-Daszkiewicz, J. et al. 2004, A\&\&A, 415, 251

Dziembowski, W. A. \& Pamiatnykh, A. A. 1993a, MNRAS, 262, 204

Dziembowski, W. A., Moskalik, P. \& Pamyatnykh, A. A. 1993b, MNRAS, 265, 588

Dziembowski, W. A. \& Pamyatnykh, A. A. 2008, MNRAS, 385, 2061

Espinosa Lara, F. \& Rieutord, M. 2007, A\& A, 470, 1013

Glatzel, W. 2009, Communications in Asteroseismology 158, 252

Godart, M., Noels, A., Dupret, M.-A., \& Lebreton, Y. 2009, MNRAS, 396, 1833

Grevesse, N. \& Noels, A. 1993, in: Hauck B. \& Paltani S. R. D. (eds.), La Formation des Eléments Chimiques, AVCP (Lausanne), p. 205

Handler, G. 2008, Communications in Asteroseismology 157, 106

Handler, G., Matthews, J. M., Eaton, J. A., Daszyńska-Daszkiewicz, J. et al. 2009, ApJ (Letters), 698, L56

Hubrig, S., Briquet, M., Schöller, M., \& De Cat, P. et al. 2006, MNRAS, 369, L61

Jackson, S., MacGregor, K. B., \& Skumanich, A. 2005, ApJS, 156, 245

Karoff, C., Arentoft, T., Glowienka, L., \& Coutures, C. et al. 2008, MNRAS, 386, 1085

Lebreton, Y., Montalbán, J., Godart, M., \& Morel, P. et al. 2009, Communications in Asteroseismology 158, 277

Lee, U. \& Saio, H. 1993, MNRAS, 261, 415

Lee, U. \& Baraffe, I. 1995, A\& A, 301, 419

Lignières, F. \& Georgeot, B. 2009, $A \& A, 500,1173$

Lovekin, C. C. \& Goupil, M.-J. 2010, A\&A, 515A, 58

Mathis, S., Palacios, A., \& Zahn, J.-P. 2004, A\&A, 425, 243

Miglio, A., Montalbán, J., \& Dupret, M.-A. 2007a, MNRAS, 375, L21

Miglio, A., Montalbán, J., Noels, A., \& Eggenberger, P. 2008, MNRAS, 386, 1487

Montalbán, J., Miglio, A., Eggenberger, P., \& Noels, A. 2008, AN, 329, 535

Noels, A., Montalban, J., Miglio, A., \& Godart, M. et al. 2010, Ap\&SS, 328, 227 
Pamyatnykh, A. A., Handler, G., \& Dziembowski, W. A. 2004, MNRAS, 350, 1022

Reese, D., Lignières, F., \& Rieutord, M. 2006, A\&A A, 455, 621

Reese, D. R., MacGregor, K. B., Jackson, S., \& Skumanich, A. et al. 2009, A\&A, 506, 189

Rogers, F. J. \& Iglesias, C. A. 1992, ApJS, 79, 507

Roxburgh, I. W. 2004, $A \& A, 428,171$

Roxburgh, I. W. 2006, $A \& A 4454,883$

Saio, H., Baker, N. H., \& Gautschy, A. 1998, MNRAS, 294, 622

Saio, H., Cameron, C., Kuschnig, R., \& Walker, G. A. H. et al. 2007, ApJ, 654, 544

Salmon, S., Montalbán, J., Miglio, A., \& Morel, T. et al. 2009, in: J. A. Guzik \& P. A. Bradley (eds.), Stellar pulsation: challenges for theory and observation, AIP-CP 1170, p. 385

Seaton, M. J. 2005, MNRAS, 362, L1

Telting, J. H. 2008, Communications in Asteroseismology 157, 112

Talon, S. 2008, in: C. Charbonnel \& J.-P. Zahn (eds.), EAS Publications Series 32, p. 81

Townsend, R. H. D. 2003, MNRAS, 343, 125

Townsend, R. H. D. 2005, MNRAS, 360, 465

Watson, R. D. 1988, ApESSS, 140, 255

Zahn, J.-P. 1991, A\&AA, 252, 179 